\title{
A 45-year-old man with cough and a cavitary lung lesion
}

Tasaduq Fazili MD

\section{CASE PRESENTATION}

$\mathrm{A}^{4}$ 45-year-old man with no significant medical history was admitted to hospital with a four-month history of intermittent fever and a predominantly dry cough. Approximately one month before admission, he started developing worsening cough with scant expectoration and pleuritic left-sided chest pain. His symptoms persisted and he presented for admission. On examination, the patient appeared chronically ill and nontoxic. His vital signs were stable except for mild tachycardia, with a heart rate in the low one hundreds. Examination of the mouth revealed him to be partially edentulous with poor orodental hygiene, although there was no evidence of dental abscesses. A chest examination revealed diminished breath sounds to the left infraaxillary area. His laboratory results were significant for mild leukocytosis (white blood cell count $13 \times 10^{9} / \mathrm{L}$ ) and mild anemia (hemoglobin level $90 \mathrm{~g} / \mathrm{L}$ ). Kidney and liver function tests were within normal limits. A chest radiograph showed a left perihilar infiltrate. An axial computed tomography (CT) scan of the chest showed a patchy infiltrate to the posterior upper lung with a central air cavity measuring $2.9 \mathrm{~cm} \times 0.7 \mathrm{~cm}$ and a small left-sided pleural effusion (Figure 1). The patient was placed on moxifloxacin and clindamycin and a procedure was performed. What is your diagnosis?

\section{Diagnosis}

The patient did not improve clinically after 10 days of antibiotic therapy and was taken to the operating room for an open left thoracotomy with decortication and drainage of the empyema. The Gram stain of the pleural fluid culture showed branching, Gram-positive rods (Figure 2) and cultures yielded Actinomyces meyeri. The patient's antibiotic regimen was switched to intravenous penicillin, three million units every $4 \mathrm{~h}$. He was treated with intravenous penicillin for a twoweek course in the hospital and was discharged home to complete a 12 -month course of oral penicillin, $500 \mathrm{mg}$ every $6 \mathrm{~h}$.

\section{DISCUSSION}

Actinomycosis is a chronic infection caused by organisms from the genus Actinomyces. Actinomyces israelii is the most commonly isolated species in humans. Other species have been implicated as being pathogenic to humans, which include Actinomyces naeslundii, Actinomyces viscosus, Actinomyces odontolyticus and A meyeri. All of these Actinomyces spieces are part of the normal oral flora and are not found in the environment.

Actinomycosis can manifest as one of three classic presentations. Oral-cervicofacial is the most common presentation, accounting for approximately $55 \%$ of cases (1). Abdominal disease occurs in $20 \%$ of patients (1), while pulmonary involvement has been reported in approximately $15 \%$ of patients (2). A meyeri is an uncommon cause of actinomycosis in humans. A review of the English language literature revealed only 26 documented cases of infection (3). The male to female ratio was approximately $3: 1$, while the mean age was approximately 43 years. More than two-thirds of the patients had evidence of gingival or dental infection and nearly one-half had a history of alcohol use.

Unlike A israelii, which predominantly causes cervicofacial disease, the most common organ system involved by A meyeri is the pulmonary system. Almost one-half of the patients experience pneumonia, with a sizeable fraction of these developing empyema (3). The reason for this

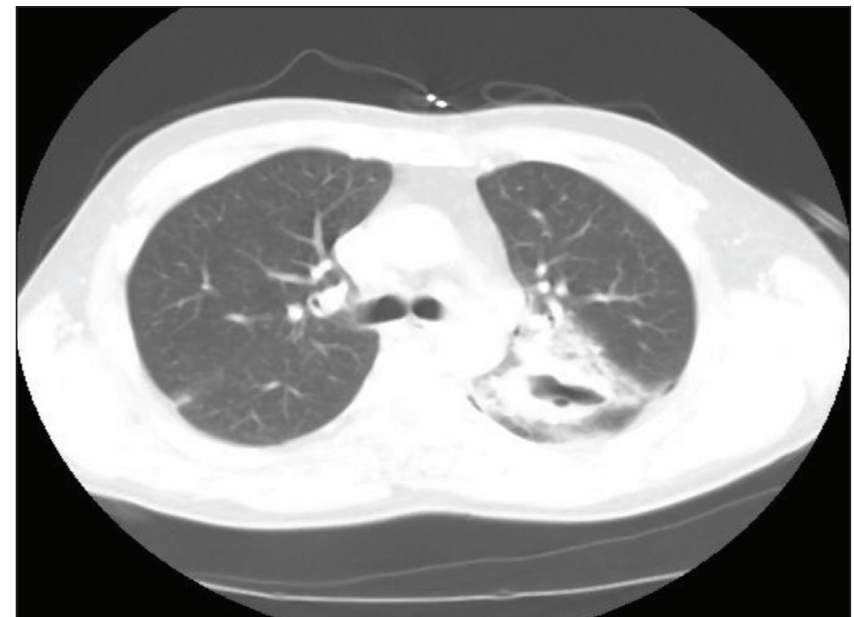

Figure 1) Computed tomography scan of the chest showing patchy infiltrate of the left lung with a central cavity

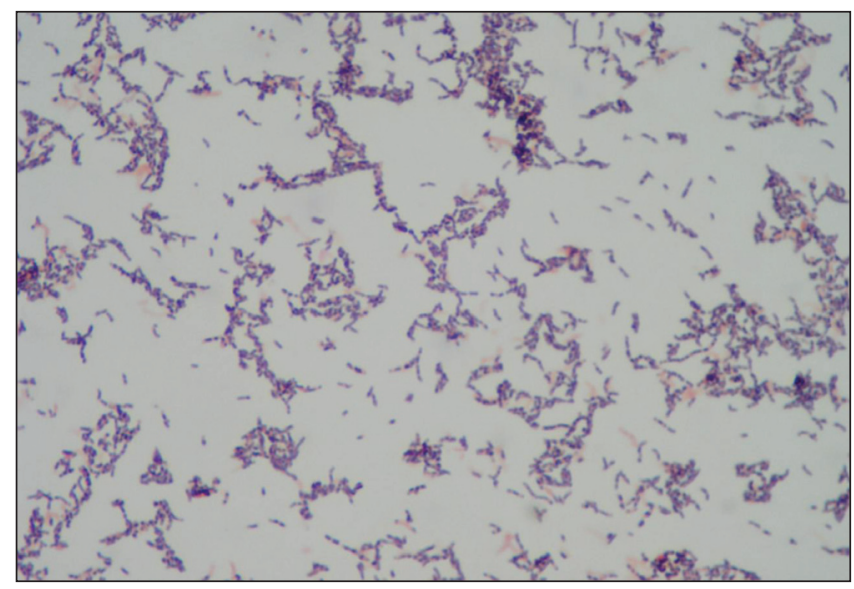

Figure 2) Gram-stain of pleural fluid culture showing branching Grampositive rods

is unclear at this time. More than one-third of patients with infection due to A meyeri have disseminated disease, and the majority of these patients have pulmonary disease (3). The exact etiology for dissemination is not known but has been postulated to be secondary to the higher rate of pulmonary involvement and subsequent bacteremia. Hematogenous spread may also occur from the oral cavity without pulmonary involvement. The most frequent areas of dissemination are the skin, long bones, liver, brain and muscles.

The one condition that can mimic actinomycosis is nocardiosis. The diagnosis of actinomycosis is definitively confirmed in the microbiology laboratory. The presence of 'sulphur granules' on hematoxylineosin staining of tissue is highly suggestive. Nocardia species do not

Department of Medicine/Infectious Disease, State University of New York Upstate University, Syracuse, New York, USA

Correspondence: Dr Tasaduq Fazili, Department of Medicine/Infectious Disease, State University of New York Upstate University,

725 Irving Avenue, Syracuse, New York 13210, USA. Telephone 315-464-9360, email fazilit@upstate.edu 
form sulphur granules. A Gram-stained specimen of infection due to Nocardia species is essentially indistinguishable from actinomycosis, and both present as filamentous Gram-positive rods. However, Nocardia species are modified acid-fast stain positive while Actinomyces species are not. In addition, Actinomyces species (especially A meyeri) grow almost exclusively anaerobically while Nocardia species grow aerobically.

Treatment in most patients with actinomycosis, including actinomycosis due to A meyeri, involves a prolonged course of antibiotics, along with surgical or percutaneous drainage, whenever indicated. Penicillin continues to be the drug of choice for Actinomyces species, including A meyeri. For localized disease, especially of the cervicofacial region, a relatively short two-month course of oral penicillin $(500 \mathrm{mg}$ to $1 \mathrm{~g}$ every $6 \mathrm{~h}$ ) is believed to be adequate. For more extensive and disseminated disease, current expert opinion suggests intravenous penicillin ( 18 million to 24 million units per day) for a period of two to six weeks, followed by oral penicillin or amoxicillin (500 mg every
$6 \mathrm{~h}$ ) for a six- to 12 -month course (4). For patients that are allergic to or intolerant of penicillin, the options include erythromycin $(500 \mathrm{mg}$ every $6 \mathrm{~h}$ ), tetracycline (500 mg every $6 \mathrm{~h})$, doxycycline (100 mg every $12 \mathrm{~h}$ ) or clindamycin ( $300 \mathrm{mg}$ to $450 \mathrm{mg}$ every $6 \mathrm{~h}$ ). Erythromycin is a reasonable alternative for the pregnant patient allergic to penicillin. The prognosis of actinomycosis is fair, even in patients with disseminated disease.

\section{REFERENCES}

1. Bennhoff D. Actinomycosis: Diagnostic and therapeutic considerations and a review of 32 cases. Laryngoscope 1984;94:1198-1217.

2. Mabeza GF, MacFarlane J. Pulmonary actinomycosis. Eur Resp J 2003;21:545-51.

3. Apothéloz C, Regamey C. Disseminated infection due to Actinomyces meyeri: Case report and review. Clin Infect Dis 1996;22:621-25.

4. Russo T. Agents of actinomycosis. In: Mandell, Douglas and Bennett's Principles and Practice of Infectious Diseases, Vol 2, 7th edn. Philadelphia: Churchill Livingstone Elsevier, 2010:3209-19. 


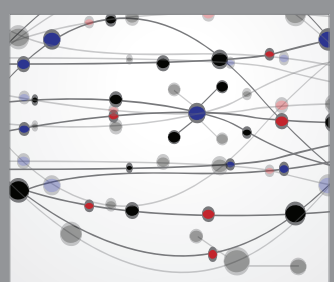

The Scientific World Journal
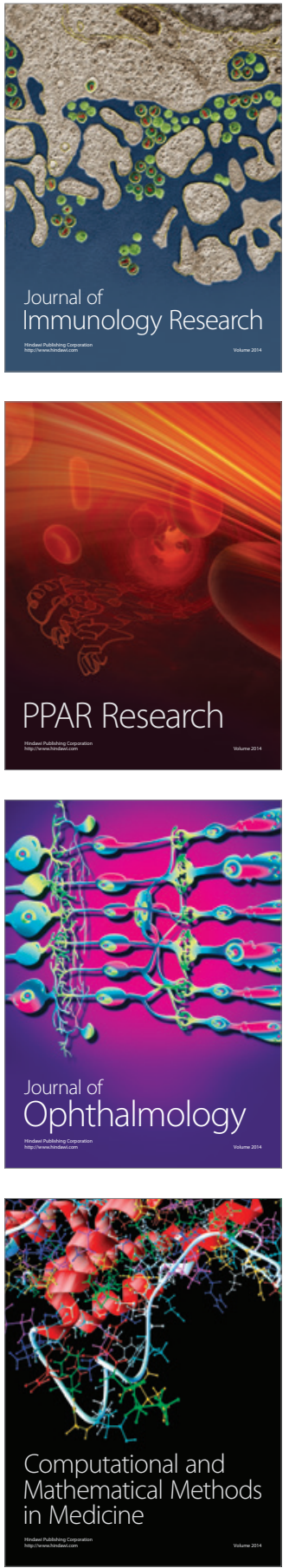

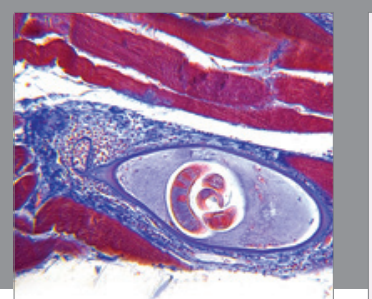

Gastroenterology Research and Practice

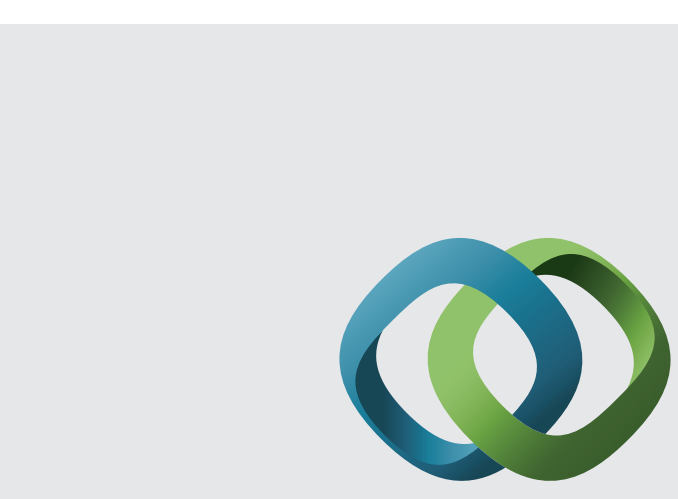

\section{Hindawi}

Submit your manuscripts at

http://www.hindawi.com
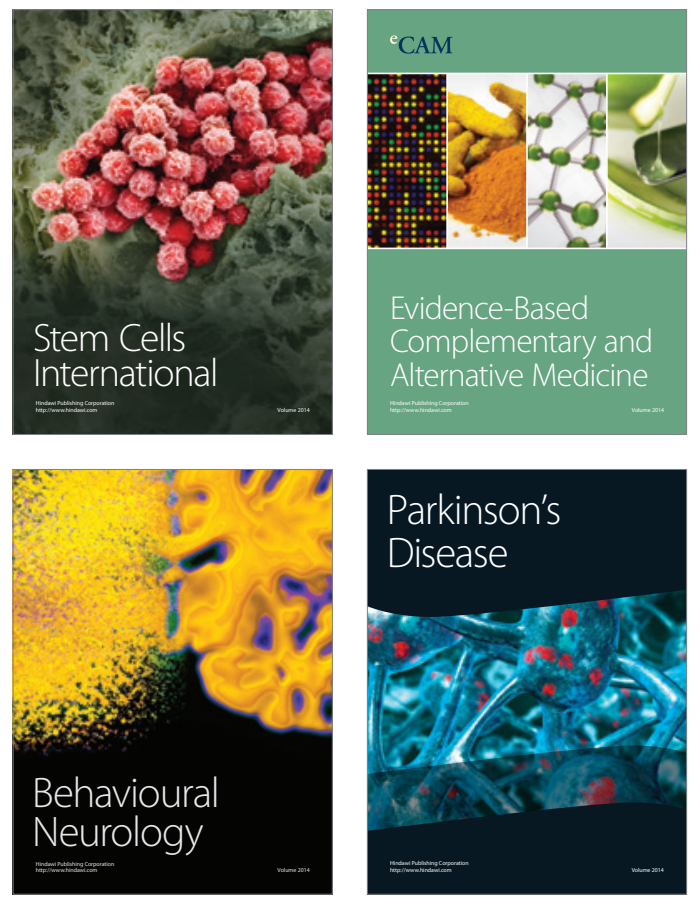
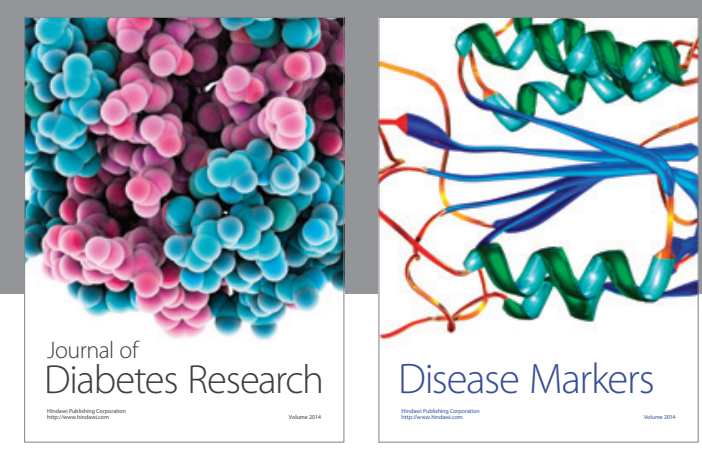

Disease Markers
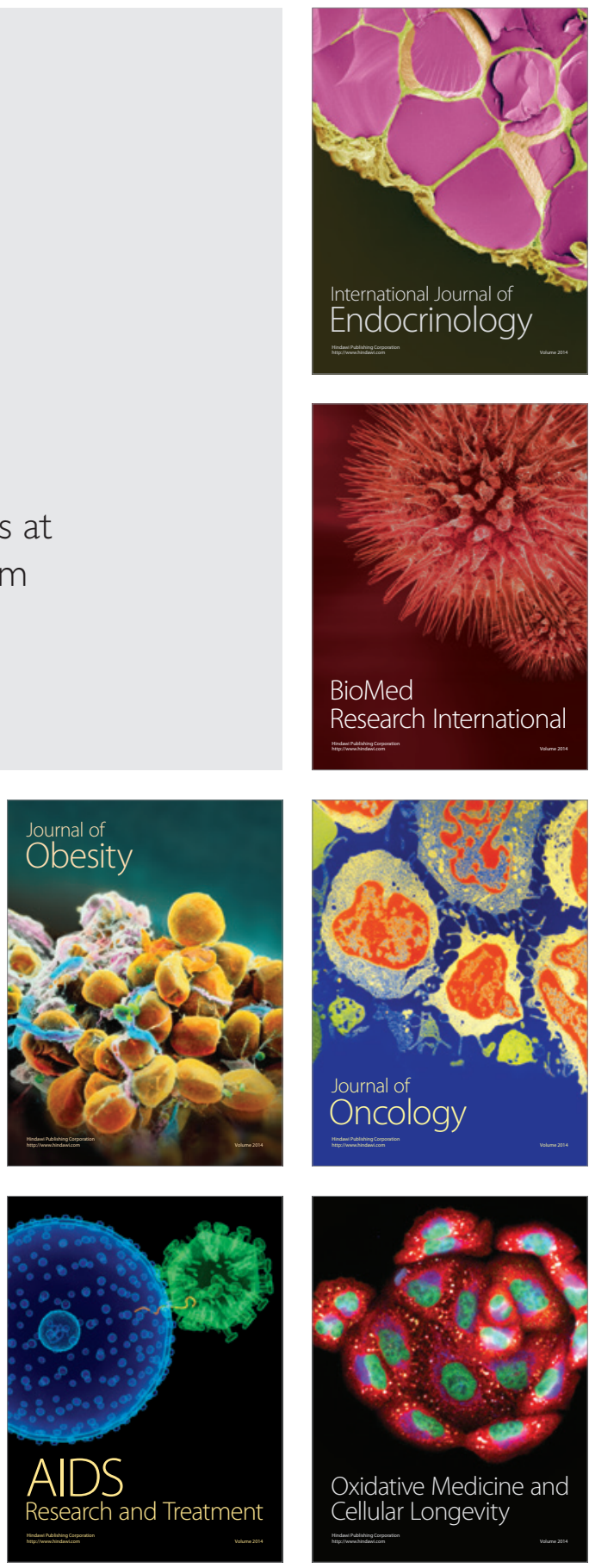